\title{
ESTUDIO TEÓRICO DE LA ACTIVIDAD ANTIOXIDANTE DE COMPUESTOS AROMÁTICOS CON BASES DE SCHIFF HETEROCÍCLICAS SUSTITUIDOS EN POSICIONES META Y PARA
}

\author{
Williams García ${ }^{\mathrm{a}}$, Hélmer Lezama ${ }^{\mathrm{a}}$, Rodolfo Pumachagua ${ }^{\mathrm{a}^{*}}$
}

\begin{abstract}
RESUMEN
En el presente estudio se analiza la relación que presenta 11 compuestos aromáticos con bases de Schiff heterocíclicas y sustituidos en posición meta y para con la actividad antioxidante, aplicando el método UB3LYP/6-311G(d,p) dentro de la teoría del funcional de densidad. Determinamos los parámetros: entalpía de disociación de enlace (BDE), potencial de ionización (IP), entalpía de disociación de protones (PDE), afinidad de protones (PA), entalpía de transferencia de electrones (ETE) y energía de estabilización de radicales (RSE), todos en fase gaseosa. Los resultados muestran que los sustituyentes electrodonadores aumentan BDE cuando se encuentran en posición para, IP en posición meta y para y ETE en posición para. Además, los electroatractores causan un aumento en PDE y PA en posición meta y para.
\end{abstract}

Palabras clave: Bases de Schiff, entalpia de disociación de enlace, potencial de ionización, entalpia de transferencia de electrones, afinidad protónica y entalpia de disociación de protón.

\section{THEORETICAL STUDY OF THE ANTIOXIDANT ACTIVITY OF AROMATIC COMPOUNDS WITH HETEROCYCLIC SCHIFF BASES SUBSTITUTED IN META AND PARA POSITIONS}

\begin{abstract}
This study analyzes the relationship that presents 11 aromatic compounds with heterocyclic Schiff bases and substituted in position meta and para with antioxidant activity, using the method UB3LYP/6-311G(d,p) within density functional theory. We determine the parameters: bond dissociation enthalpy (BDE), ionization potential (IP), protons dissociation enthalpy (PDE), affinity of protons (PA), electrons transfer enthalpy (ETE) and radical stabilization energy (RSE), all in gaseous phase. The results show that electrodonors substituents increases BDE when they are in position para, IP in position meta and para and ETE in position para. In addition, the electroatractors cause an increase in PDE and PA at position meta and para.

a Universidad Nacional Federico Villarreal, Facultad de Ciencias Naturales y Matemática, Laboratorio de Química Teórica, Jr. Chepén s/n (El Agustino) Perú.*rpumachagua@gmail.com
\end{abstract}


Key words: Schiff bases, bond dissociation enthalpy, ionization potential, electrons transfer enthalpy, proton affinity and proton dissociation enthalpy.

\section{INTRODUCCIÓN}

Las bases de Schiff constituyen un grupo importante de compuestos orgánicos que tienen un amplio rango de aplicabilidad en diversos campos de la química y biología. La actividad de las bases de Schiff se da en los sistemas biológicos como antioxidantes, antiinflamatorios, analgésicos, antimicrobianos, anticonvulsivos, antituberculosos, anticancerosos y antihelmínticos, además del papel como precursores en la síntesis de diferentes compuestos orgánicos de importancia biológica. Se cree que el átomo de nitrógeno del grupo imina puede estar implicado en la formación de enlaces de hidrógeno con los centros activos de los constituyentes de las células y los efectos en los procesos celulares normales ${ }^{1}$. Estos son considerados como "ligandos privilegiados" y son muy utilizados debido a su síntesis versátil y buena solubilidad. ${ }^{2}$

Los métodos de la teoría del funcional de densidad (DFT), se utilizaron para evaluar las propiedades químicas y dilucidar la relación estructura-actividad (SAR) de compuestos aromáticos con bases de Schiff heterocíclicas con posible actividad antioxidante. Además, el estudio de las propiedades electrónicas y moleculares será de gran importancia para comprender el mecanismo de la actividad antioxidante.

\section{FUNDAMENTO TEÓRICO}

\section{Actividad antioxidante}

Los antioxidantes presentan la capacidad de ceder sus electrones a los radicales libres estabilizando la estructura de estos e inhibiendo el proceso de oxidación causada y de esta forma neutraliza sus efectos dañinos..$^{3-4}$

Los compuestos fenólicos y sus derivados tienen gran potencial antioxidante debido a sus grupos hidroxilo fenólicos que pueden actuar como donantes de hidrógeno o de electrones, eliminando los radicales libres.

Los mecanismos a través de los cuales los antioxidantes pueden neutralizar los radicales libres (esquema 1$)^{5}$ son principalmente: (1) la transferencia de átomos de hidrógeno (HAT), (2) la transferencia de electrones única seguida de transferencia de protones (ET-PT) y (3) la transferencia de electrones seguida de pérdida de protones (SPLET). 


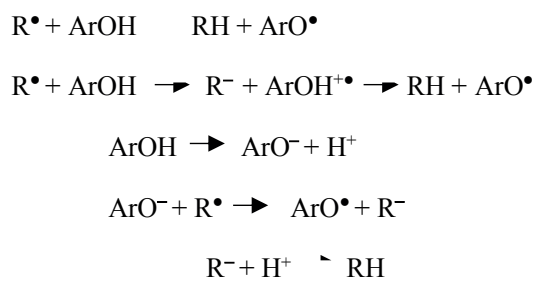

Esquema 1. Mecanismos a través de los cuales los antioxidantes neutralizan los radicales libres.

En el mecanismo (1) la reactividad de $\mathrm{ArOH}$ se estima calculando BDE de O-H, cuanto menor es su valor, mayor es la actividad esperada. En el mecanismo (2) el primer paso es una reacción de transferencia de electrones, cuyo parámetro de control es IP de ArOH; se espera que las moléculas con valores IP más bajos tengan una actividad más alta. PDE está involucrado en el mecanismo (3), donde ETE de $\mathrm{ArO}^{-}$es otro parámetro de control. Por lo tanto, $(\mathrm{BDE})^{6,7},(\mathrm{IP}),(\mathrm{PDE}),(\mathrm{PA})^{8}$ y $(\mathrm{ETE})$ se utilizarán como principales descriptores moleculares para elucidar la actividad de eliminación de radicales en los compuestos.

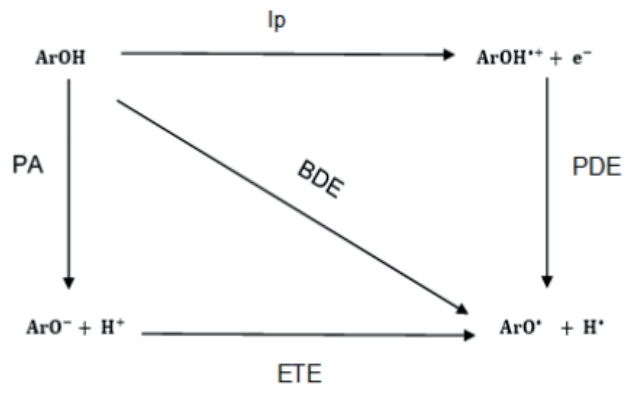

Figura 1. Esquema de los mecanismos antioxidantes y los parámetros que los definen.

\section{Efecto sustituyente}

Los grupos sustituyentes ejercen los efectos más importantes que influyen en las propiedades químicas, físicas y biológicas de una especie química, estos se describen con frecuencia mediante varias constantes del sustituyente y están relacionados con sus propiedades electrónicas y del sitio de reacción. ${ }^{9-11}$

La ecuación de Hammett y sus formas extendidas han sido uno de los medios más utilizados para el estudio e interpretación de reacciones orgánicas y sus mecanismos ${ }^{12,13}$. Hammett propuso la existencia de una relación entre la velocidad de reacción y la constante de equilibrio. Como resultado, se ha encontrado la correlación de la constante de Hammett $(\sigma)$ y los grupos sustituyentes. ${ }^{14}$ Los grupos electroatractores tendrán valores positivos de $\sigma$ y los electrodonadores valores negativos. A medida que sea más alta la magnitud de $\sigma$ mayor será su capacidad electrónica. ${ }^{15}$ 


\section{ASPECTOS COMPUTACIONALES}

Todos los cálculos se realizaron en el programa Gaussian 09. Las estructuras se optimizaron con restricción de simetría utilizando el funcional híbrido UB3LYP y la función base 6-311G(d,p ${ }^{16}$. Se procedió a encontrar los parámetros termodinámicos: BDE, IP, PDE, PA y ETE usando las siguientes ecuaciones:

$$
\begin{gathered}
\mathrm{BDE}=\mathrm{H}\left(\mathrm{I}_{\mathrm{m}}^{\bullet}\right)-\mathrm{H}\left(\mathrm{H}^{\bullet}\right)+\mathrm{H}\left(\mathrm{I}_{\mathrm{m}}\right) \\
\mathrm{PDE}=\mathrm{H}\left(\mathrm{I}_{\mathrm{m}}^{\bullet}\right)+\mathrm{H}\left(\mathrm{H}^{+}\right)-\mathrm{H}\left(\mathrm{I}_{\mathrm{m}}{ }^{+}\right) \\
\mathrm{PI}=\mathrm{H}\left(\mathrm{I}_{\mathrm{m}}^{+}\right)-\mathrm{H}\left(\mathrm{I}_{\mathrm{m}}^{\bullet}\right) \\
\mathrm{PA}=\mathrm{H}\left(\mathrm{I}_{\mathrm{m}}^{-}\right)+\mathrm{H}\left(\mathrm{H}^{+}\right)-\mathrm{H}\left(\mathrm{I}_{\mathrm{m}}^{+}\right) \\
\mathrm{ETE}=\mathrm{H}\left(\mathrm{I}_{\mathrm{m}}^{\bullet}\right)-\mathrm{H}\left(\mathrm{I}_{\mathrm{m}}{ }^{-}\right) \\
\mathrm{RSE}=\mathrm{H}\left(\mathrm{I}_{\mathrm{m}}\right)+\mathrm{H}\left(\mathrm{OH}^{\bullet}\right)-\mathrm{H}\left(\mathrm{I}_{\mathrm{m}}^{\bullet}\right)-\mathrm{H}\left(\mathrm{H}_{2} \mathrm{O}\right)
\end{gathered}
$$

dónde: $\mathrm{Im}_{\mathrm{Im}} \bullet \mathrm{Im}^{+}, \mathrm{Im}^{-}, \mathrm{H}^{\bullet}, \mathrm{H}^{+}, \mathrm{OH}^{\bullet}$ y $\mathrm{H}_{2} \mathrm{O}$ son las entalpias del compuesto aromático con base de Schiff heterocíclica: base, radical, catión y anión; radical hidrógeno, protón, radical hidroxilo, y molécula de agua, respectivamente.

\section{RESULTADOS Y DISCUSIÓN}

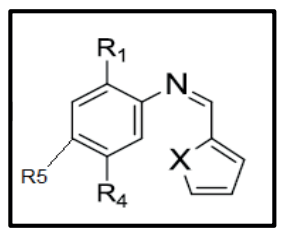

Figura 2. Estructura base aromática con base de Schiff heterocíclica. 
Tabla 1. Sistemas aromáticos con bases de Schiff heterocíclicas sustituidos.

\begin{tabular}{|c|c|c|}
\hline Sistema & Grupos sustituyen & \\
\hline $\mathbf{I}_{0}$ & $\mathrm{X}=\mathrm{NH} \quad \mathrm{R}_{1}=\mathrm{OH} \quad \mathrm{R}_{4}=\mathrm{H}$ & $\mathrm{R}_{5}=\mathrm{H}$ \\
\hline $\mathbf{I}_{1}$ & $\mathrm{X}=\mathrm{NH} \quad \mathrm{R}_{1}=\mathrm{OH} \quad \mathrm{R}_{4}=\mathrm{CH}_{3}$ & $\mathrm{R}_{5}=\mathrm{H}$ \\
\hline $\mathbf{I}_{2}$ & $\mathrm{X}=\mathrm{NH} \quad \mathrm{R}_{1}=\mathrm{OH} \quad \mathrm{R}_{4}=\mathrm{H}$ & $\mathrm{R}_{5}=\mathrm{CH}_{3}$ \\
\hline $\mathbf{I}_{4}$ & $\mathrm{X}=\mathrm{NH} \quad \mathrm{R}_{1}=\mathrm{OH} \quad \mathrm{R}_{4}=\mathrm{H}$ & $\mathrm{R}_{5}=\mathrm{CH}_{3} \mathrm{O}$ \\
\hline $\mathbf{I}_{6}$ & $\mathrm{X}=\mathrm{NH} \quad \mathrm{R}_{1}=\mathrm{OH} \quad \mathrm{R}_{4}=\mathrm{H}$ & $\mathrm{R}_{5}=\mathrm{NO}_{2}$ \\
\hline $\mathbf{I}_{7}$ & $\mathrm{X}=\mathrm{NH} \quad \mathrm{R}_{1}=\mathrm{OH} \quad \mathrm{R}_{4}=\mathrm{CH}_{3} \mathrm{CO}$ & $\mathrm{R}_{5}=\mathrm{H}$ \\
\hline $\mathbf{I}_{8}$ & $\mathrm{X}=\mathrm{NH} \quad \mathrm{R}_{1}=\mathrm{OH} \quad \mathrm{R}_{4}=\mathrm{H}$ & $\mathrm{R}_{5}=\mathrm{CH}_{3} \mathrm{CO}$ \\
\hline $\mathbf{I}_{9}$ & $\mathrm{X}=\mathrm{NH} \quad \mathrm{R}_{1}=\mathrm{OH} \quad \mathrm{R}_{4}=\mathrm{NH}_{2}$ & $\mathrm{R}_{5}=\mathrm{H}$ \\
\hline $\mathbf{I}_{10}$ & $\mathrm{X}=\mathrm{NH} \quad \mathrm{R}_{1}=\mathrm{OH} \quad \mathrm{R}_{4}=\mathrm{H}$ & $\mathrm{R}_{5}=\mathrm{NH}_{2}$ \\
\hline $\mathbf{I}_{11}$ & $\mathrm{X}=\mathrm{NH} \quad \mathrm{R}_{1}=\mathrm{OH} \quad \mathrm{R}_{4}=\mathrm{CN}$ & $\mathrm{R}_{5}=\mathrm{H}$ \\
\hline $\mathbf{I}_{12}$ & $\mathrm{X}=\mathrm{NH} \quad \mathrm{R}_{1}=\mathrm{OH} \quad \mathrm{R}_{4}=\mathrm{H}$ & $\mathrm{R}_{5}=\mathrm{CN}$ \\
\hline $\mathbf{I}_{13}$ & $\mathrm{X}=\mathrm{NH} \quad \mathrm{R}_{1}=\mathrm{OH} \quad \mathrm{R}_{4}=\mathrm{CF}_{3}$ & $\mathrm{R}_{5}=\mathrm{H}$ \\
\hline $\mathbf{I}_{14}$ & $\mathrm{X}=\mathrm{NH} \quad \mathrm{R}_{1}=\mathrm{OH} \quad \mathrm{R}_{4}=\mathrm{H}$ & $\mathrm{R}_{5}=\mathrm{CF}_{3}$ \\
\hline $\mathbf{I}_{15}$ & $\mathrm{X}=\mathrm{NH} \quad \mathrm{R}_{1}=\mathrm{OH} \quad \mathrm{R}_{4}=\mathrm{F}$ & $\mathrm{R}_{5}=\mathrm{H}$ \\
\hline $\mathbf{I}_{16}$ & $\mathrm{X}=\mathrm{NH} \quad \mathrm{R}_{1}=\mathrm{OH} \quad \mathrm{R}_{4}=\mathrm{H}$ & $\mathrm{R}_{5}=\mathrm{F}$ \\
\hline $\mathbf{I}_{17}$ & $\mathrm{X}=\mathrm{NH} \quad \mathrm{R}_{1}=\mathrm{OH} \quad \mathrm{R}_{4}=\mathrm{Cl}$ & $\mathrm{R}_{5}=\mathrm{H}$ \\
\hline $\mathbf{I}_{18}$ & $\mathrm{X}=\mathrm{NH} \quad \mathrm{R}_{1}=\mathrm{OH} \quad \mathrm{R}_{4}=\mathrm{H}$ & $\mathrm{R}_{5}=\mathrm{Cl}$ \\
\hline $\mathbf{I}_{19}$ & $\mathrm{X}=\mathrm{NH} \quad \mathrm{R}_{1}=\mathrm{OH} \quad \mathrm{R}_{4}=\mathrm{Br}$ & $\mathrm{R}_{5}=\mathrm{H}$ \\
\hline $\mathbf{I}_{20}$ & $\mathrm{X}=\mathrm{NH} \quad \mathrm{R}_{1}=\mathrm{OH} \quad \mathrm{R}_{4}=\mathrm{H}$ & $\mathrm{R}_{5}=\mathrm{Br}$ \\
\hline $\mathbf{I}_{21}$ & $\mathrm{X}=\mathrm{NH} \quad \mathrm{R}_{1}=\mathrm{OH} \quad \mathrm{R}_{4}=\mathrm{OH}$ & $\mathrm{R}_{5}=\mathrm{H}$ \\
\hline $\mathbf{I}_{22}$ & $\mathrm{X}=\mathrm{NH} \quad \mathrm{R}_{1}=\mathrm{OH} \quad \mathrm{R}_{4}=\mathrm{H}$ & $\mathrm{R}_{5}=\mathrm{OH}$ \\
\hline
\end{tabular}

Tabla 2. Valores BDE, $\triangle \mathrm{BDE}$ y $\sigma \mathrm{m}, \mathrm{p}$ en compuestos aromáticos con base de Schiff heterocíclicas meta y para sustituidos.

\begin{tabular}{|c|c|c|c|c|}
\hline Sistema & Sustituyente & $\mathrm{BDE}(\mathrm{kJ} / \mathrm{mol})$ & $\Delta \mathrm{BDE}(\mathrm{kJ} / \mathrm{mol})$ & $\sigma_{\mathrm{m}, \mathrm{p}}^{12}$ \\
\hline & & Fase gas & Fase gas & \\
\hline $\mathbf{I}_{0}$ & & 90,7 & & \\
\hline$I_{9}$ & $p-\mathrm{NH}_{2}$ & 84,5 & $-6,1$ & $-0,66$ \\
\hline $\mathbf{I}_{21}$ & $p-\mathrm{OH}$ & 88,2 & $-2,5$ & $-0,37$ \\
\hline $\mathbf{I}_{3}$ & $p-\mathrm{CH}_{3} \mathrm{O}$ & 88,0 & $-2,6$ & $-0,27$ \\
\hline $\mathbf{I}_{\mathbf{1}}$ & $p-\mathrm{CH}_{3}$ & 89,4 & $-1,3$ & $-0,17$ \\
\hline $\mathbf{I}_{10}$ & $m-\mathrm{NH}_{2}$ & 91,9 & 1,2 & $-0,16$ \\
\hline $\mathbf{I}_{2}$ & $m-\mathrm{CH}_{3}$ & 91,0 & 0,3 & $-0,07$ \\
\hline$I_{15}$ & $p-\mathrm{F}$ & 90,2 & $-0,4$ & 0,06 \\
\hline$I_{4}$ & $m-\mathrm{CH}_{3} \mathrm{O}$ & 90,9 & 0,3 & 0,12 \\
\hline $\mathbf{I}_{22}$ & $m-\mathrm{OH}$ & 92,2 & 1,5 & 0,12 \\
\hline $\mathbf{I}_{17}$ & $p-\mathrm{Cl}$ & 90,8 & 0,1 & 0,23 \\
\hline$I_{19}$ & $p-\mathrm{Br}$ & 90,7 & 0,0 & 0,23 \\
\hline $\mathbf{I}_{16}$ & $m-\mathrm{F}$ & 91,8 & 1,2 & 0,34 \\
\hline$I_{18}$ & $m-\mathrm{Cl}$ & 91,5 & 0,8 & 0,37 \\
\hline$I_{8}$ & $m-\mathrm{CH}_{3} \mathrm{CO}$ & 91,8 & 1,1 & 0,38 \\
\hline $\mathbf{I}_{20}$ & $m-\mathrm{Br}$ & 91,8 & 0,3 & 0,39 \\
\hline$I_{14}$ & $m-\mathrm{CF}_{3}$ & 92,1 & 1,5 & 0,43 \\
\hline $\mathbf{I}_{7}$ & $p-\mathrm{CH}_{3} \mathrm{CO}$ & 93,5 & 2,8 & 0,5 \\
\hline $\mathbf{I}_{13}$ & $p-\mathrm{CF}_{3}$ & 93,5 & 1,6 & 0,54 \\
\hline$I_{12}$ & $m-\mathrm{CN}$ & 92,3 & 1,6 & 0,56 \\
\hline$I_{11}$ & $p-\mathrm{CN}$ & 92,5 & $-0,2$ & 0,66 \\
\hline$I_{6}$ & $m-\mathrm{NO}_{2}$ & 92,3 & 1,6 & 0,71 \\
\hline$I_{5}$ & $p-\mathrm{NO}_{2}$ & 94,6 & 1,5 & 0,78 \\
\hline
\end{tabular}




\section{Dependencia de $\triangle \mathrm{BDE}$ con la constante de Hammett}

$\Delta \mathrm{BDE}=\mathrm{BDE}\left(\mathrm{I}_{\mathrm{X}}\right)-\mathrm{BDE}\left(\mathrm{I}_{0}\right)$

IX: sistema sustituido, $\mathrm{I}_{0}$ : sistema base.

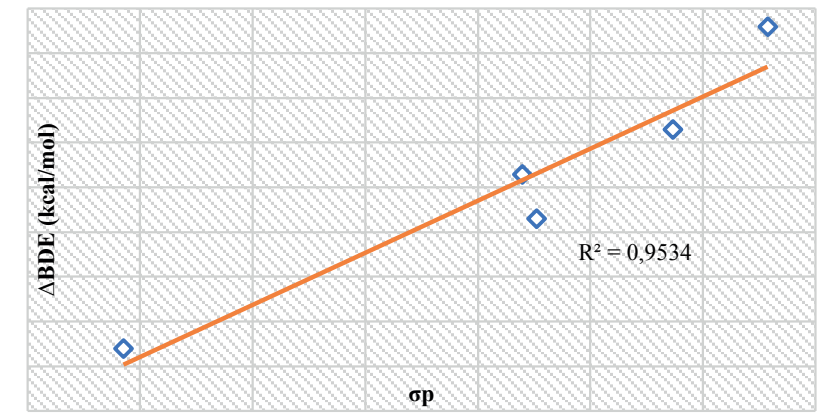

Figura 3. Dependencia de $\triangle \mathrm{BDE}$ con $\sigma_{\mathrm{p}}$.

Los valores calculados BDE y $\triangle \mathrm{BDE}$ en compuestos aromáticos con base de Schiff heterocíclicas meta y para sustituidos se muestran en la tabla 2. El efecto del sustituyente en la posición para es más significativo en los sistemas: $\mathbf{I}_{\mathbf{9}}, \mathbf{I}_{3}, \mathbf{I}_{\mathbf{1}}$ y $\mathbf{I}_{\mathbf{2 1}}$. Los valores más bajos están relacionados con grupos fuertes donantes de electrones como: $-\mathrm{NH}_{2},-\mathrm{CH}_{3} \mathrm{O},-\mathrm{CH}_{3}$ y - $\mathrm{OH}$ respectivamente. Los valores más altos de BDE están relacionados con grupos fuertes extractores de electrones como $-\mathrm{NO}_{2}$ en el sistema $\mathbf{I}_{5}$.

Los grupos halógenos: $-\mathrm{F}$ y $-\mathrm{Br}$ en los sistemas: $\mathbf{I}_{15}$ y $\mathbf{I}_{19}$ tienen efectos opuestos sobre la molécula base y el radical. Ellos pueden desestabilizar la molécula base aumentando su energía y estabilizando la fracción radical por efecto de resonancia. Estos dos efectos opuestos dan como resultado valores BDE reducidos en presencia de átomos de halógeno en comparación con otros grupos extractores de electrones.

En la figura 3 se representa la correlación entre la constante de Hammett $\left(\sigma_{\mathrm{p}}\right)$ con valores $\triangle \mathrm{BDE}$ calculadas. La ecuación de la regresión lineal es la siguiente:

$$
\Delta \mathrm{BDE}(\mathrm{kcal} / \mathrm{mol})=0,1164 \sigma \mathrm{p}+0,0192
$$

La linealidad es satisfactoria, cuando se realiza para los sistemas: $\mathbf{I}_{1}, \mathbf{I}_{3}, \mathbf{I}_{\mathbf{9}}, \mathbf{I}_{15}, \mathbf{I}_{19}$ y $\mathbf{I}_{\mathbf{2 1}}$.

\section{Dependencia de $\Delta$ IP con la constante de Hammett}

$$
\Delta \mathrm{IP}=\mathrm{IP}(\mathrm{IX})-\mathrm{IP}\left(\mathrm{I}_{0}\right)
$$


Tabla 3. Valores IP, $\triangle \mathrm{IP}$ y $\sigma \mathrm{m}, \mathrm{p}$ en compuestos aromáticos con base de Schiff heterocíclicas meta y para sustituidos.

\begin{tabular}{|c|c|c|c|c|}
\hline Sistema & Sustituyente & $\mathrm{IP}(\mathrm{kJ} / \mathrm{mol})$ & $\Delta \mathrm{IP}(\mathrm{kJ} / \mathrm{mol})$ & $\sigma_{\mathrm{m}, \mathrm{p}}{ }^{12}$ \\
\hline $\mathbf{I}_{0}$ & & $\begin{array}{c}\text { Fase gas } \\
169,9\end{array}$ & Fase gas & \\
\hline $\mathbf{I}_{9}$ & $p-\mathrm{NH}_{2}$ & 155,4 & $-14,5$ & $-0,66$ \\
\hline $\mathbf{I}_{21}$ & $p-\mathrm{OH}$ & 165,8 & $-4,1$ & $-0,37$ \\
\hline $\mathbf{I}_{3}$ & $p-\mathrm{CH}_{3} \mathrm{O}$ & 163,6 & $-6,3$ & $-0,27$ \\
\hline $\mathbf{I}_{1}$ & $p-\mathrm{CH}_{3}$ & 166,5 & $-3,4$ & $-0,17$ \\
\hline $\mathbf{I}_{10}$ & $m-\mathrm{NH}_{2}$ & 153,7 & $-16,2$ & $-0,16$ \\
\hline $\mathbf{I}_{2}$ & $m-\mathrm{CH}_{3}$ & 166,3 & $-3,6$ & $-0,07$ \\
\hline $\mathbf{I}_{15}$ & $p-\mathrm{F}$ & 173,8 & 3,8 & 0,06 \\
\hline $\mathbf{I}_{22}$ & $m-\mathrm{OH}$ & 165,9 & $-4,0$ & 0,12 \\
\hline $\mathbf{I}_{4}$ & $m-\mathrm{CH}_{3} \mathrm{O}$ & 161,5 & $-8,4$ & 0,12 \\
\hline $\mathbf{I}_{17}$ & $p-\mathrm{Cl}$ & 174,2 & 4,3 & 0,23 \\
\hline $\mathbf{I}_{19}$ & $p-\mathrm{Br}$ & 172,4 & 2,4 & 0,23 \\
\hline $\mathbf{I}_{16}$ & $m-\mathrm{F}$ & 174,0 & 4,1 & 0,34 \\
\hline $\mathbf{I}_{18}$ & $m-\mathrm{Cl}$ & 174,1 & 4,2 & 0,37 \\
\hline $\mathbf{I}_{8}$ & $m-\mathrm{CH}_{3} \mathrm{CO}$ & 175,4 & 5,4 & 0,38 \\
\hline $\mathbf{I}_{20}$ & $m-\mathrm{Br}$ & 172,6 & 2,7 & 0,39 \\
\hline $\mathbf{I}_{14}$ & $m-\mathrm{CF}_{3}$ & 181,1 & 11,2 & 0,43 \\
\hline $\mathbf{I}_{7}$ & $p-\mathrm{CH}_{3} \mathrm{CO}$ & 174,0 & 4,0 & 0,50 \\
\hline $\mathbf{I}_{13}$ & $p-\mathrm{CF}_{3}$ & 180,7 & 10,8 & 0,54 \\
\hline $\mathbf{I}_{12}$ & $m-\mathrm{CN}$ & 179,4 & 9,5 & 0,56 \\
\hline $\mathbf{I}_{11}$ & $p-\mathrm{CN}$ & 179,4 & 9,5 & 0,66 \\
\hline $\mathbf{I}_{6}$ & $m-\mathrm{NO}_{2}$ & 183,6 & 13,7 & 0,71 \\
\hline $\mathbf{I}_{5}$ & $p-\mathrm{NO}_{2}$ & 182,8 & 12,9 & 0,78 \\
\hline
\end{tabular}

Los valores IP en compuestos aromáticos con base de Schiff heterocíclicas meta y para sustituidos se presentan en la tabla 3 . Observamos que los sistemas: $\mathbf{I}_{1}, \mathbf{I}_{2}, \mathbf{I}_{3}, \mathbf{I}_{4}, \mathbf{I}_{9}, \mathbf{I}_{10}, \mathbf{I}_{21}$ y $\mathbf{I}_{22}$ poseen valores IP más bajos respecto al sistema base. Cuanto menor es el valor IP, más fácil será la transferencia de electrones en el mecanismo de transferencia de electrones individuales y mayor será la actividad antioxidante.

El grupo sustituyente $m-\mathrm{NH}_{2}$ el cual es buen donador de electrones permitiría la transferencia del electrón, dando así mayor actividad antioxidante. Por el contrario, con el sustituyente $m-\mathrm{NO}_{2}$, un extractor de electrones, la transferencia del electrón no podría ocurrir, por tanto el valor de IP sería muy alto. 


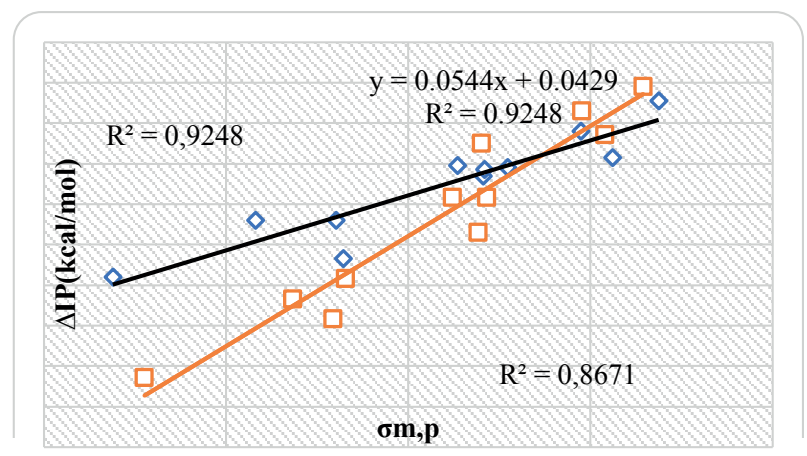

Figura 4. Dependencia de $\Delta \mathrm{IP}$ con $\sigma_{\mathrm{m}}$ (línea negra) y $\sigma_{\mathrm{p}}$ (línea anaranjada).

En la figura 4 se representa la correlación que hay entre las constantes de Hammett $\left(\sigma_{\mathrm{m}, \mathrm{p}}\right)$ con valores $\Delta I P$ calculadas. Las ecuaciones obtenidas son las siguientes:

$$
\begin{aligned}
& \Delta \mathrm{IP}=0,0544 \sigma_{\mathrm{m}}+0,0429 \\
& \Delta \mathrm{IP}=0,0272 \sigma_{\mathrm{p}}+0,2439
\end{aligned}
$$

Los coeficientes de correlación, $\mathrm{R}^{2}$, alcanzaron valores de 0,92 y 0,87 para los compuestos aromáticos con base de Schiff heterocíclicas meta y para sustituidos, respectivamente. La linealidad es aceptable, y pueden predecir la disminución del IP en compuestos aromáticos con base de Schiff heterocíclicas meta y para sustituidas a partir de sus constantes de Hammett o viceversa.

\section{Dependencia de las $\triangle P D E$ con las constantes de Hammett}

$$
\triangle \mathrm{PDE}=\mathrm{PDE}(\mathrm{IX})-\mathrm{PDE}\left(\mathrm{I}_{0}\right)
$$

En el mecanismo de transferencia de electrones seguida de protones, el electrón se produce a partir del antioxidante para formar un anión que transfiere un protón. La transferencia del electrón depende de IP, mientras que la del protón se rige por el valor PDE. Cuanto menor sea el valor de PDE, más fácil será la transferencia de protones del catión radical del sistema $\left(\mathrm{I}_{\mathrm{X}}{ }^{+}\right)$ $\mathrm{y}$, por tanto, más estable será su radical $\left(\mathrm{I}_{\mathrm{X}}^{\bullet}\right)$ formado, siendo mayor la actividad antioxidante. 
Tabla 4. Valores PDE, $\triangle \mathrm{PDE}$ y $\sigma_{\mathrm{m}, \mathrm{p}}$ en compuestos aromáticos con base de Schiff heterocíclicas meta y para sustituidos.

\begin{tabular}{|c|c|c|c|c|}
\hline Sistema & Sustituyente & PDE (kJ /mol) & $\Delta \mathrm{PDE}(\mathrm{kJ} / \mathrm{mol})$ & $\sigma_{\mathrm{m}, \mathrm{p}}{ }^{12}$ \\
\hline & & Fase gas & Fase gas & \\
\hline $\mathbf{I}_{0}$ & & 234,7 & & \\
\hline I9 & $p-\mathrm{NH}_{2}$ & 243,0 & 8,3 & $-0,66$ \\
\hline $\mathbf{I}_{21}$ & $p-\mathrm{OH}$ & 236,3 & 1,6 & $-0,37$ \\
\hline $\mathbf{I}_{3}$ & $p-\mathrm{CH}_{3} \mathrm{O}$ & 238,4 & 3,7 & $-0,27$ \\
\hline $\mathbf{I}_{\mathbf{1}}$ & $p-\mathrm{CH}_{3}$ & 236,9 & 2,2 & $-0,17$ \\
\hline $\mathbf{I}_{10}$ & $m-\mathrm{NH}_{2}$ & 252,1 & 17,4 & $-0,16$ \\
\hline $\mathbf{I}_{2}$ & $m-\mathrm{CH}_{3}$ & 238,6 & 3,9 & $-0,07$ \\
\hline $\mathbf{I}_{15}$ & $p-\mathrm{F}$ & 230,4 & $-4,3$ & 0,06 \\
\hline $\mathbf{I}_{22}$ & $m-\mathrm{OH}$ & 240,2 & 5,5 & 0,12 \\
\hline $\mathbf{I}_{4}$ & $m-\mathrm{CH}_{3} \mathrm{O}$ & 243,3 & 8,6 & 0,12 \\
\hline $\mathbf{I}_{17}$ & $p-\mathrm{Cl}$ & 230,5 & $-4,2$ & 0,23 \\
\hline $\mathbf{I}_{19}$ & $p-\mathrm{Br}$ & 232,2 & $-2,4$ & 0,23 \\
\hline $\mathbf{I}_{16}$ & $m-\mathrm{F}$ & 231,8 & $-2,9$ & 0,34 \\
\hline $\mathbf{I}_{18}$ & $m--\mathrm{Cl}$ & 231,3 & $-3,4$ & 0,37 \\
\hline $\mathbf{I}_{8}$ & $m-\mathrm{CH}_{3} \mathrm{CO}$ & 230,3 & $-4,3$ & 0,38 \\
\hline $\mathbf{I}_{20}$ & $m-\mathrm{Br}$ & 233,1 & $-1,6$ & 0,39 \\
\hline $\mathbf{I}_{14}$ & $m-\mathrm{CF}_{3}$ & 224,9 & $-9,7$ & 0,43 \\
\hline $\mathbf{I}_{7}$ & $p-\mathrm{CH}_{3} \mathrm{CO}$ & 233,5 & $-1,2$ & 0,5 \\
\hline $\mathbf{I}_{13}$ & $p-\mathrm{CF}_{3}$ & 226,7 & $-7,9$ & 0,54 \\
\hline $\mathbf{I}_{12}$ & $m-\mathrm{CN}$ & 226,8 & $-7,9$ & 0,56 \\
\hline $\mathbf{I}_{11}$ & $p-\mathrm{CN}$ & 227,0 & $-7,7$ & 0,66 \\
\hline $\mathbf{I}_{6}$ & $m-\mathrm{NO}_{2}$ & 222,6 & $-12,1$ & 0,71 \\
\hline
\end{tabular}

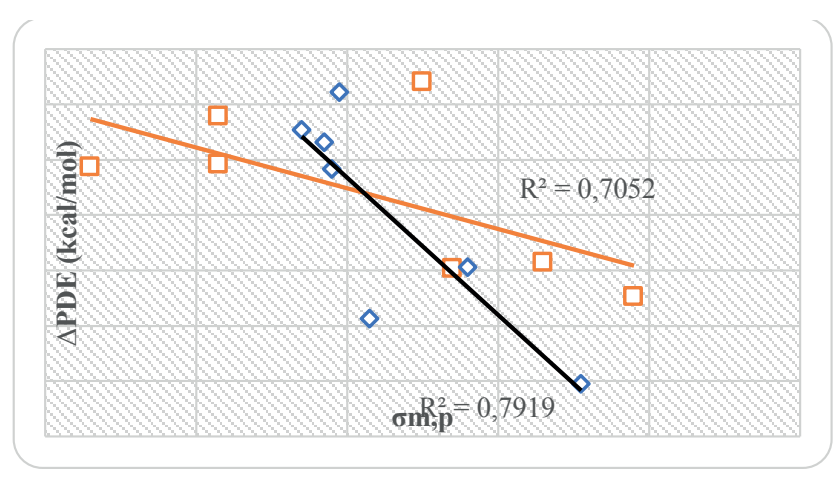

Figura 5. Dependencia de $\Delta \mathrm{PDE}$ con $\sigma_{\mathrm{m}}$ (línea negra) y $\sigma_{\mathrm{p}}$ (línea anaranjada). 
En la tabla 4, observamos que los sistemas: $\mathbf{I}_{5}, \mathbf{I}_{6}, \mathbf{I}_{7}, \mathbf{I}_{8}, \mathbf{I}_{11}, \mathbf{I}_{12}, \mathbf{I}_{13}, \mathbf{I}_{14}, \mathbf{I}_{15}, \mathbf{I}_{16}, \mathbf{I}_{17}, \mathbf{I}_{18}, \mathbf{I}_{19} \mathrm{y}$ $\mathbf{I}_{20}$ tienen valores de PDE más bajos, siendo $\mathbf{I}_{6}$ el que mejor valor nos dio comparado con el sistema base $\left(\mathbf{I}_{0}\right)$, mostrando mayor posibilidad de transferencia de protones (PDE). Entonces si relacionamos los valores PDE de la tabla 2 con los IP de la tabla 3, podemos ver que los sistemas: $\mathbf{I}_{1}, \mathbf{I}_{2}, \mathbf{I}_{3}, \mathbf{I}_{4}, \mathbf{I}_{9}, \mathbf{I}_{10}, \mathbf{I}_{21}$ y $\mathbf{I}_{22}$ mostraron valores de IP muy bajos, no seguirían el mecanismo de transferencia de protones.

En la figura 5 se representa la correlación que hay entre las constantes de Hammett $\left(\sigma_{\mathrm{m}, \mathrm{p}}\right)$ con valores $\triangle \mathrm{PDE}$ calculados. Las ecuaciones obtenidas son las siguientes:

$$
\begin{aligned}
\Delta \mathrm{PDE} & =-24,811 \sigma_{\mathrm{m}}+5,2781 \\
\Delta \mathrm{PDE} & =-7,3636 \sigma_{\mathrm{p}}-2,0844
\end{aligned}
$$

Los coeficientes de correlación, $\mathrm{R}^{2}$, alcanzaron valores de 0,8 y 0,7 para los compuestos aromáticos con base de Schiff heterocíclicas meta y para sustituidos, respectivamente.

\section{Dependencia de las $\triangle \mathrm{PA}$ con las constantes de Hammett}

$$
\Delta \mathrm{PA}=\mathrm{PA}\left(\mathrm{I}_{\mathrm{X}}\right)-\mathrm{PA}\left(\mathrm{I}_{0}\right)
$$

\begin{tabular}{|c|c|c|c|c|}
\hline Sistema & Sustituyente & $\mathrm{PA}(\mathrm{kJ} / \mathrm{mol})$ & $\Delta \mathrm{PA}(\mathrm{kJ} / \mathrm{mol})$ & $\sigma_{\mathrm{m}, \mathrm{p}}{ }^{12}$ \\
\hline & & Fase gas & Fase gas & \\
\hline $\mathbf{I}_{0}$ & & 359 & 0 & \\
\hline I9 & $p-\mathrm{NH}_{2}$ & 365 & 6 & $-0,66$ \\
\hline $\mathbf{I}_{21}$ & $p-\mathrm{OH}$ & 361 & 2 & $-0,37$ \\
\hline $\mathbf{I}_{3}$ & $p-\mathrm{CH}_{3} \mathrm{O}$ & 361 & 2 & $-0,27$ \\
\hline $\mathbf{I}_{\mathbf{1}}$ & $p-\mathrm{CH}_{3}$ & 359 & 0 & $-0,17$ \\
\hline $\mathbf{I}_{10}$ & $m-\mathrm{NH}_{2}$ & 363 & 4 & $-0,16$ \\
\hline $\mathbf{I}_{2}$ & $m-\mathrm{CH}_{3}$ & 359 & 0 & $-0,07$ \\
\hline $\mathbf{I}_{15}$ & $p-\mathrm{F}$ & 355 & -4 & 0,06 \\
\hline $\mathbf{I}_{22}$ & $m-\mathrm{OH}$ & 359 & 0 & 0,12 \\
\hline $\mathbf{I}_{4}$ & $m-\mathrm{CH}_{3} \mathrm{O}$ & 359 & 0 & 0,12 \\
\hline $\mathbf{I}_{17}$ & $p-\mathrm{Cl}$ & 352 & -7 & 0,23 \\
\hline $\mathbf{I}_{19}$ & $p-\mathrm{Br}$ & 352 & -7 & 0,23 \\
\hline $\mathbf{I}_{16}$ & $m-\mathrm{F}$ & 353 & -6 & 0,34 \\
\hline $\mathbf{I}_{18}$ & $m-\mathrm{Cl}$ & 350 & -9 & 0,37 \\
\hline Is & $m-\mathrm{CH}_{3} \mathrm{CO}$ & 353 & -6 & 0,38 \\
\hline $\mathbf{I}_{20}$ & $m-\mathrm{Br}$ & 351 & -8 & 0,39 \\
\hline $\mathbf{I}_{14}$ & $m-\mathrm{CF}_{3}$ & 347 & -12 & 0,43 \\
\hline $\mathbf{I}_{7}$ & $p-\mathrm{CH}_{3} \mathrm{CO}$ & 348 & -11 & 0,5 \\
\hline $\mathbf{I}_{13}$ & $p-\mathrm{CF}_{3}$ & 345 & -14 & 0,54 \\
\hline $\mathbf{I}_{12}$ & $m-\mathrm{CN}$ & 347 & -12 & 0,56 \\
\hline $\mathbf{I}_{11}$ & $p-\mathrm{CN}$ & 344 & -15 & 0,66 \\
\hline $\mathbf{I}_{6}$ & $m-\mathrm{NO}_{2}$ & 344 & -15 & 0,71 \\
\hline $\mathbf{I}_{5}$ & $p-\mathrm{NO}_{2}$ & 337 & -22 & 0,78 \\
\hline
\end{tabular}

Tabla 5. Valores PA, $\triangle \mathrm{PA}$ y $\sigma_{\mathrm{m}, \mathrm{p}}$ en compuestos aromáticos con base de Schiff heterocíclicas meta y para sustituidos. 
El parámetro PA mide la facilidad de eliminación del protón en compuestos aromáticos con base de Schiff heterocíclicas meta y para sustituidos ( $\mathrm{I}_{\mathrm{x}}$ ) para formar su forma aniónica ( $\mathrm{I}_{\mathrm{x}}^{-}$). Los sistemas: $\mathbf{I}_{5}, \mathbf{I}_{6}, \mathbf{I}_{7}, \mathbf{I}_{8}, \mathbf{I}_{11}, \mathbf{I}_{12}, \mathbf{I}_{13}, \mathbf{I}_{14}, \mathbf{I}_{15}, \mathbf{I}_{16}, \mathbf{I}_{17}, \mathbf{I}_{18}, \mathbf{I}_{19}$ y $\mathbf{I}_{20}$ tienen un valor PA más bajo que el sistema base $\left(\mathbf{I}_{0}\right)$. El sistema $\mathbf{I}_{5}$ tiene el valor más bajo por tener un sustituyente extractor de electrones, generando así buena actividad antioxidante. Sin embargo, existen algunas excepciones, tal es el caso del sistema $\mathbf{I}_{9}$ que posee al sustituyente $p-\mathrm{NH}_{2}$, buen donante de electrones cuyo valor PA es más alto que $\mathbf{I}_{0}$.

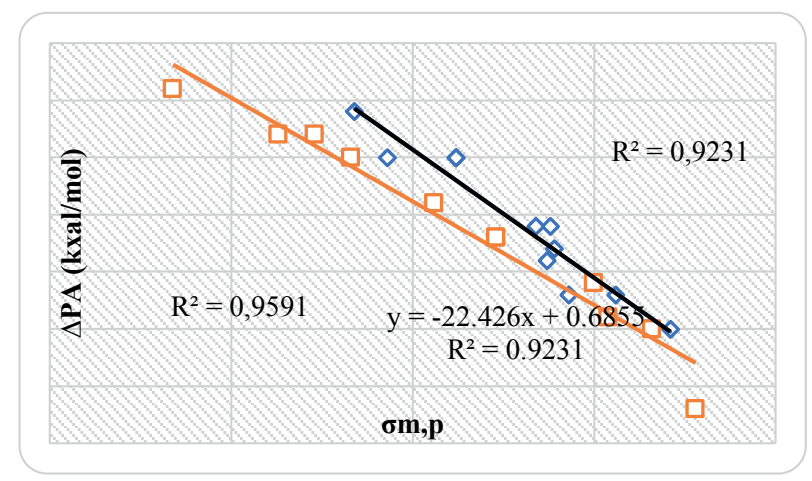

Figura 6. Dependencia de $\Delta \mathrm{PA}$ con $\sigma_{\mathrm{m}}$ (línea negra) y $\sigma_{\mathrm{p}}$ (línea anaranjada).

En la figura 6 se representa la correlación que hay entre las constantes de $\operatorname{Hammett}\left(\sigma_{\mathrm{m}, \mathrm{p}}\right)$ con valores $\triangle \mathrm{PA}$ calculadas. Las ecuaciones obtenidas son las siguientes:

$$
\begin{gathered}
\Delta \mathrm{PA}=-22,426 \sigma_{\mathrm{m}}+0,6855 \\
\Delta \mathrm{PA}=-18,124 \sigma_{\mathrm{p}}-3,8428
\end{gathered}
$$

Los coeficientes de correlación, $\mathrm{R}^{2}$, alcanzan valores de 0,92 y 0,96 para los compuestos aromáticos con base de Schiff heterocíclicas meta y para sustituidos, respectivamente. La linealidad encontrada es satisfactoria y puede predecir la disminución de PA en los sistemas meta y para sustituidos a partir de sus constantes de Hammett o viceversa.

\section{Dependencia de las $\triangle \mathrm{ETE}$ con la constante de Hammett}

$$
\Delta \mathrm{ETE}=\mathrm{ETE}(\mathrm{IX})-\mathrm{ETE}\left(\mathrm{I}_{0}\right)
$$

En el mecanismo SPLET, los parámetros como PA y ETE juegan un papel importante. Con el fin de eliminar fácilmente el protón de los sistemas meta y para sustituidos $\left(\mathrm{I}_{\mathrm{x}}\right)$ para obtener su forma aniónica $\left(\mathrm{I}_{\mathrm{x}}^{-}\right)$, la PA debe ser más baja. De forma similar, la transferencia de electrones del sistema aniónico $\left(\mathrm{I}_{\mathrm{x}}^{-}\right)$para obtener su forma radicalaria $\left(\mathrm{I}_{\mathrm{x}}{ }^{\bullet}\right)$ será más fácil, cuando tengan el valor ETE más bajo. 
A partir de la tabla 6 observamos que los sistemas: $\mathbf{I}_{\mathbf{1}}, \mathbf{I}_{\mathbf{3}}, \mathbf{I}_{\mathbf{9}}, \mathbf{I}_{\mathbf{1 0}}$ y $\mathbf{I}_{\mathbf{2}}$ tienen un valor ETE más bajo respecto al sistema base $\left(\mathbf{I}_{\mathbf{0}}\right)$. Esto significa que la transferencia de electrones será más fácil. Por lo tanto, los sistemas meta y para sustituidos tienen valores más bajos de PA y ETE, lo que indica una mayor posibilidad de someterse al mecanismo SPLET para la eliminación de radicales, mostrando mayor actividad antioxidante, pero si relacionamos los valores ETE de la tabla 6 con PA de la tabla 5, observamos que los sistemas: $\mathbf{I}_{5}, \mathbf{I}_{6}, \mathbf{I}_{7}, \mathbf{I}_{\mathbf{8}}, \mathbf{I}_{11}, \mathbf{I}_{12}, \mathbf{I}_{13}, \mathbf{I}_{14}, \mathbf{I}_{15}$, $\mathbf{I}_{16}, \mathbf{I}_{17}, \mathbf{I}_{18}, \mathbf{I}_{19} \mathrm{y}_{\mathbf{I}_{20}}$ tienen valores PA muy bajos, no seguirían el mecanismo de transferencia de electrón (ETE).

Tabla 6. Valores de ETE, $\triangle$ ETE y $\sigma_{\mathrm{m}, \mathrm{p}}$ en compuestos aromáticos con base de Schiff heterocíclicas y con sustituyentes en posiciones meta y para.

\begin{tabular}{|c|c|c|c|c|}
\hline Sistema & Sustituyente & ETE $(\mathrm{kcal} / \mathrm{mol})$ & $\Delta \mathrm{ETE}(\mathrm{kcal} / \mathrm{mol})$ & $\sigma_{\mathrm{m}, \mathrm{p}}{ }^{12}$ \\
\hline & & Fase gas & Fase gas & \\
\hline $\mathbf{I}_{0}$ & & 45,5 & 0 & \\
\hline $\mathbf{I}_{9}$ & $p-\mathrm{NH}_{2}$ & 33,9 & $-11,7$ & $-0,66$ \\
\hline $\mathbf{I}_{3}$ & $p-\mathrm{CH}_{3} \mathrm{O}$ & 40,8 & $-4,8$ & $-0,27$ \\
\hline $\mathbf{I}_{21}$ & $p-\mathrm{OH}$ & 41,4 & $-4,1$ & $-0,37$ \\
\hline $\mathbf{I}_{1}$ & $p-\mathrm{CH}_{3}$ & 44,0 & $-1,5$ & $-0,17$ \\
\hline $\mathbf{I}_{10}$ & $m-\mathrm{NH}_{2}$ & 42,4 & $-3,1$ & $-0,16$ \\
\hline $\mathbf{I}_{2}$ & $m-\mathrm{CH}_{3}$ & 45,6 & 0,0 & $-0,07$ \\
\hline $\mathbf{I}_{15}$ & $p-\mathrm{F}$ & 48,9 & 3,4 & 0,06 \\
\hline $\mathbf{I}_{22}$ & $m-\mathrm{OH}$ & 47,5 & 2,0 & 0,12 \\
\hline $\mathbf{I}_{4}$ & $m-\mathrm{CH}_{3} \mathrm{O}$ & 46,2 & 0,7 & 0,12 \\
\hline $\mathbf{I}_{17}$ & $p-\mathrm{Cl}$ & 52,9 & 7,4 & 0,23 \\
\hline $\mathbf{I}_{19}$ & $p-\mathrm{Br}$ & 52,1 & 6,6 & 0,23 \\
\hline $\mathbf{I}_{16}$ & $m-\mathrm{F}$ & 52,8 & 7,3 & 0,34 \\
\hline $\mathbf{I}_{18}$ & $m-\mathrm{Cl}$ & 55,1 & 9,6 & 0,37 \\
\hline Is & $m-\mathrm{CH}_{3} \mathrm{CO}$ & 52,9 & 7,4 & 0,38 \\
\hline $\mathbf{I}_{20}$ & $m-\mathrm{Br}$ & 54,6 & 9,1 & 0,39 \\
\hline $\mathbf{I}_{14}$ & $m-\mathrm{CF}_{3}$ & 59,0 & 13,5 & 0,43 \\
\hline $\mathbf{I}_{7}$ & $p-\mathrm{CH}_{3} \mathrm{CO}$ & 59,2 & 13,7 & 0,5 \\
\hline $\mathbf{I}_{13}$ & $p-\mathrm{CF}_{3}$ & 62,0 & 16,6 & 0,54 \\
\hline $\mathbf{I}_{12}$ & $m-\mathrm{CN}$ & 59,2 & 13,7 & 0,56 \\
\hline $\mathbf{I}_{11}$ & $p-\mathrm{CN}$ & 62,3 & 16,8 & 0,66 \\
\hline $\mathbf{I}_{6}$ & $m-\mathrm{NO}_{2}$ & 61,9 & 16,4 & 0,71 \\
\hline $\mathbf{I}_{5}$ & $p-\mathrm{NO}_{2}$ & 71,2 & 25,7 & 0,78 \\
\hline
\end{tabular}




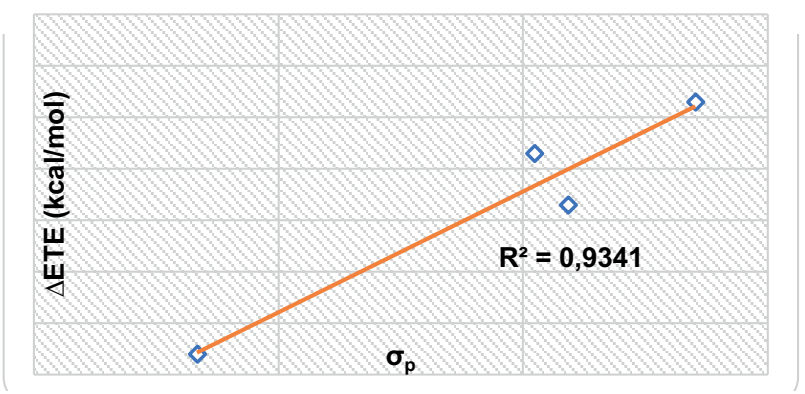

Figura 7. Dependencia de las $\Delta$ ETE con la $\sigma_{\mathrm{p}}$.

En la figura 7 se representa la correlación que hay entre la constante de $\operatorname{Hammett}\left(\sigma_{\mathrm{p}}\right)$ con valores $\triangle E T E$ calculadas.

La ecuación obtenida es la siguiente:

$$
\Delta \mathrm{ETE}=0,047 \sigma_{\mathrm{p}}-0,1093
$$

El coeficiente de correlación, $\mathrm{R}^{2}$, alcanzo el valor de 0,93 para los compuestos aromáticos con base de Schiff heterocíclicas para sustituidos. La linealidad es satisfactoria para 4 sistemas: $\mathbf{I}_{1}, \mathbf{I}_{3}, \mathbf{I}_{9}, \mathrm{y} \mathbf{I}_{21}$.

Relación de RSE en compuestos aromáticos con bases de Schiff heterocíclicas sustituidos en posición meta y para con valores BDE, ETE y PDE. 
Tabla 7. Valores RSE, BDE, ETE y PDE compuestos aromáticos con bases de Schiff heterocíclicas y con sustituyentes en posiciones meta y para.

\begin{tabular}{|c|c|c|c|c|c|}
\hline Sistema & Sustituyente & $\begin{array}{c}\text { BDE }(\mathrm{kcal} / \mathrm{mol}) \\
\text { Fase gas }\end{array}$ & $\begin{array}{c}\text { ETE }(\mathrm{kcal} / \mathrm{mol}) \\
\text { Fase gas }\end{array}$ & $\begin{array}{c}\text { PDE }(\mathrm{kcal} / \mathrm{mol}) \\
\text { Fase gas }\end{array}$ & $\begin{array}{c}\text { RSE }(\mathrm{kcal} / \mathrm{mol}) \\
\text { Fase gas }\end{array}$ \\
\hline $\mathbf{I}_{0}$ & & 90,7 & 45,5 & 234,7 & 15,8 \\
\hline $\mathbf{I}_{9}$ & $p-\mathrm{NH}_{2}$ & 84,5 & 33,9 & 243,0 & 22,0 \\
\hline $\mathbf{I}_{3}$ & $p-\mathrm{CH}_{3} \mathrm{O}$ & 88,0 & 40,8 & 238,4 & 18,4 \\
\hline $\mathbf{I}_{1}$ & $p-\mathrm{CH}_{3}$ & 89,4 & 44,0 & 236,9 & 17,1 \\
\hline $\mathbf{I}_{10}$ & $m-\mathrm{NH}_{2}$ & 91,9 & 42,4 & 252,1 & 14,6 \\
\hline $\mathbf{I}_{15}$ & $p-\mathrm{F}$ & 90,2 & 48,9 & 230,4 & 16,2 \\
\hline $\mathbf{I}_{2}$ & $m-\mathrm{CH}_{3}$ & 91,0 & 45,6 & 238,6 & 15,5 \\
\hline $\mathbf{I}_{4}$ & $m-\mathrm{CH}_{3} \mathrm{O}$ & 90,9 & 46,2 & 243,3 & 15,5 \\
\hline $\mathbf{I}_{17}$ & $p-\mathrm{Cl}$ & 90,8 & 52,9 & 230,5 & 15,7 \\
\hline $\mathbf{I}_{19}$ & $p-\mathrm{Br}$ & 90,7 & 52,1 & 232,2 & 15,8 \\
\hline $\mathbf{I}_{16}$ & $m-\mathrm{F}$ & 91,8 & 52,8 & 231,8 & 14,6 \\
\hline I8 & $m-\mathrm{CH}_{3} \mathrm{CO}$ & 91,8 & 52,9 & 230,3 & 14,7 \\
\hline $\mathbf{I}_{18}$ & $m-\mathrm{Cl}$ & 91,5 & 55,1 & 231,3 & 15,0 \\
\hline $\mathbf{I}_{20}$ & $m-\mathrm{Br}$ & 91,8 & 54,6 & 233,1 & 14,7 \\
\hline $\mathbf{I}_{14}$ & $m-\mathrm{CF}_{3}$ & 92,1 & 59,0 & 224,9 & 14,3 \\
\hline $\mathbf{I}_{7}$ & $p-\mathrm{CH}_{3} \mathrm{CO}$ & 93,5 & 59,2 & 233,5 & 13,0 \\
\hline $\mathbf{I}_{13}$ & $p-\mathrm{CF}_{3}$ & 93,5 & 62,0 & 226,7 & 13,0 \\
\hline $\mathbf{I}_{12}$ & $m-\mathrm{CN}$ & 92,3 & 59,2 & 226,8 & 14,2 \\
\hline $\mathbf{I}_{11}$ & $p-\mathrm{CN}$ & 92,5 & 62,3 & 227,0 & 14,0 \\
\hline$I_{6}$ & $m-\mathrm{NO}_{2}$ & 92,3 & 61,9 & 222,6 & 14,2 \\
\hline $\mathbf{I}_{5}$ & $p-\mathrm{NO}_{2}$ & 94,6 & 71,2 & 225,7 & 11,9 \\
\hline $\mathbf{I}_{22}$ & $m-\mathrm{OH}$ & 92,2 & 47,5 & 240,2 & 14,3 \\
\hline $\mathbf{I}_{21}$ & $p-\mathrm{OH}$ & 88,2 & 41,4 & 236,3 & 18,3 \\
\hline
\end{tabular}

La energía de estabilización de radicales (RSE) es el parámetro que indica la estabilidad del compuesto aromático con base heterocíclica y sustituido $\left(\mathrm{I}_{\mathrm{x}}\right)$ formada como radical. Cuanto mayor sea el valor RSE, más estable será la imina radicalaria y mayor será la actividad antioxidante. A partir de la tabla 7 se deduce: los sistemas: $\mathbf{I}_{9}, \mathbf{I}_{3}, \mathbf{I}_{1}$ y $\mathbf{I}_{21}$, al transferir el átomo de hidrógeno y convertirse a su forma radicalaria, cuando se mide esta pérdida a través del parámetro de BDE y ETE, estas serán más estables que el sistema base por poseer RSE más alto. El sistema I15 también cumple con este comportamiento. 


\section{CONCLUSIONES}

En la actividad antioxidante de los compuestos aromáticos con bases de Schiff heterocíclicas se comprueba para el parámetro:

1. BDE, el mecanismo de transferencia de un átomo de hidrógeno es mayor cuando están sustituidos con grupos dadores de electrones- $\pi$ en posición para.

2. IP, el mecanismo de transferencia de un electrón es mayor cuando están sustituidos con grupos dadores de electrones- $\pi$ en posición para y meta.

3. PDE, el mecanismo secuencial de transferencia de un electrón y protón es mayor cuando están sustituidos con grupos sustractores de electrones- $\pi$ en posición para y meta.

4. PA, el mecanismo de transferencia de un protón es mayor cuando están sustituidos con grupos sustractores de electrones- $\pi$ en posición para y meta.

5. ETE, el mecanismo secuencial de transferencia de un protón y electrón es mayor cuando están sustituidos con grupos dadores de electrones- $\pi$ en posición para.

Finalmente, los compuestos aromáticos con bases de Schiff heterocíclicas y sustituidos con grupos: $-\mathrm{NH}_{2},-\mathrm{CH}_{3} \mathrm{O},-\mathrm{CH}_{3} \mathrm{y}-\mathrm{OH}$, todos en posición para, fueron más estables y con alto potencial antioxidante, cuando se considera el mecanismo de transferencia del átomo de hidrógeno o la transferencia secuencial de un protón seguida de un electrón. Esto también ocurre con el grupo $p-\mathrm{F}$.

\section{REFERENCIAS BIBLIOGRÁFICAS}

1. Pandey A, Rajavel R, Chandraker S, Dash D. Synthesis of Schiff bases of 2-amino5-aryl-1,3,4-thiadiazole and its anasgesic, anti-inflammatory and anti-bacterial activity. E-J Chem. 2012; 9: 2524-2531.

2. Rafi UM, Mahendiran D, Haleel AK, Nankar RP, Doble M, Rahiman AK. New pyridazine-based binuclear nickel(II), copper(II) and zinc(II) complexes as prospective anticancer agents. New J Chem. 2016; 40: 2451-2465.

3. Son S, Lewis BA. Free radical scavenging and antioxidative activity of caffeic acid amide and ester analogues: Structure-activity relationship. J Agric Food Chem. 2002; 50: 468-472.

4. Zapata Cuartas JC. Obtención de una nueva serie de compuestos pirrilicos y estudio teórico de su potencial actividad antioxidante. [Tesis de maestría]. Medellín: Universidad Nacional de Colombia; 2012.

5. Lu L, Zhu S, Zhang H, Zhang S. Improvement of antioxidative activity of resveratrol by elongating conjugated chain: A DFT theoretical study. Comput Theor Chem. 2013; 1019: 39-47.

6. Wright JS, Johnson ER, DiLabio GA. Predicting the activity of phenolic antioxidants: Theoretical method, analysis of substituent effects, and application to major families of antioxidants. J Am Chem Soc. 2001; 123: 1173-1183. 
7. Cotes S, Cotuá J, Muñoz A. Calculated antioxidant activity of selected phenolic compounds. Can J Chem. 2018; 96: 345-350.

8. Becke AD. Density-functional thermochemistry. III. The role of exact exchange. J Chem Phys. 1993; 98: 5648-5652.

9. Sengar RS, Nemykin VN, Basu P. Electronic properties of para-substituted thiophenols and disulfides from 13C NMR spectroscopy and ab initio calculations: relations to the Hammett parameters and atomic charges. New J Chem. 2003; 27: 1115- 1123.

10. Chandra AK, Uchimaru T. The O-H bond dissociation energies of substituted phenols and proton affinities of substituted phenoxide ions: A DFT study. Int J Mol Sci. 2002; 3: 407-422.

11. Krygowski TM, Stępién BT. Sigma- and pi-electron delocalization: Focus on substituent effects. Chem Rev. 2005; 105: 3482-3512.

12. Hansch C, Leo A, Taft RW. A survey of Hammett substituent constants and resonance and field parameters. Chem Rev. 1991; 91: 165-195.

13. Exner O, Böhm S. Background of the Hammett equation as observed for isolated molecules: Meta- and para-substituted benzoic acids. J Org Chem. 2002; 67: 6320-6327.

14. McDaniel H, Brown HC. An extended table of Hammett substituent constants based on the ionization of substituted benzoic acids. J Org Chem. 1957; 23: 420-427.

15. La Macchia G, Gagliardi L, Carlson GS, Jay AN, Davis E, Cramer CJ. Theoretical prediction of linear free energy relationships using proton nucleomers. J Phys Org Chem. 2008; 21: 136-145.

16. Klein E, Lukeš V, Cibulková Z, Polovková J. Study of N-H, O-H, and S-H bond dissociation enthalpies and ionization potentials of substituted anilines, phenols, and thiophenols. J Mol Struct-THEOCHEM. 2006; 758: 149-159. 